\title{
Mortalidad de niños menores de 5 años después de la implementación de la estrategia atención integrada a las enfermedades prevalentes en la infancia (AIEPI) - Antioquia, Colombia $2002-2011$
}

\author{
Mortality of children under 5 years old after the implementation of the strategy for integrated diseases prevalent in \\ childhood (AIEPI) Antioquia, Colombia 2002 - 2011
}

Karen Sofía Quiroz-Jiménez , Lina María Pulgarín-Piedrahita², Doris Cardona-Arango ${ }^{3}$

1 Bacterióloga, Especialista en Gerencia de la Salud Pública. Profesional de apoyo Alcaldía municipal de Guatapé - Dirección Local de Salud. Guatapé, Colombia. e-mail: ksofi07@gmail.com

2 Nutricionista Dietista. Especialista en Gerencia de la Salud Pública. Alcaldía de Medellín, Unidad de Seguridad Alimentaria y Nutricional. Medellín, Colombia.e-mail: Impulgarinp@gmail.com

3 PhD. en Demografía. Docente Universidad CES. Medellín, Colombia. e-mail: dcardona@ces.edu.co

Fecha de recepción: Noviembre $3-2014$

Fecha de aceptación: Septiembre 18 - 2015

Quiroz-Jiménez KS, Pulgarín-Piedrahita LM, Cardona-Arango D. Mortalidad de niños menores de 5 años después de la implementación de la estrategia atención integrada a las enfermedades prevalentes en la infancia (AIEPI) - Antioquia, Colombia 2002 - 2011. Univ. Salud. 2017;(2): 201 - 211. DOI: http://dx.doi.org/10.22267/rus.151702.5

\begin{abstract}
Objetivo: Analizar el comportamiento de la mortalidad por enfermedad diarreica aguda, infección respiratoria aguda y desnutrición en niños menores de 5 años en las regiones del departamento de Antioquia entre 2002 y 2011, a partir de la implementación de la estrategia AIEPI. Materiales y métodos: Estudio descriptivo retrospectivo con enfoque cuantitativo de fuente secundaria, con registros de defunciones de menores de cinco años del Departamento de Antioquia 2002-2011, consignados en el Departamento Administrativo Nacional de Estadísticas; el análisis fue univariado y bivariado, con comparación de proporciones y tasas de mortalidad. Resultados: Las regiones muestran descenso en las cifras de mortalidad infantil por las tres causas, con diferencias entre ellas. Los hombres menores de un año presentaron una mayor proporción de muertes, con diferencias significativas, superiores al 80\%. Se encontró asociación entre las causas de muerte y las regiones de origen de los fallecidos ( $\mathrm{p}=0,000)$. Conclusión: Aunque hay descenso en las cifras de mortalidad en menores de cinco años, la implementación de AIEPI ha cumplido un papel importante en el control por estas causas de muerte, pero no hay evidencia que haya sido determinante para que ocurriera este descenso.
\end{abstract}

Resumen

Palabras clave: Desnutrición, niño, salud, mortalidad, AIEPI. (Fuente: DeCS, Bireme).

\begin{abstract}
Objective: To analyze the behavior of mortality from acute diarrheal disease, acute respiratory infection and malnutrition in children under 5 years old in the regions of the department of Antioquia between 2002 and 2011 by implementing the AIEPI strategy. Materials and methods: A retrospective descriptive study with quantitative approach of secondary source was made by using the records of deaths of children under five years old in the department of Antioquia between 2002 and 2011 which were listed in the National Administrative Department of statistics. The analysis was univariate and bivariate, with comparison of proportions and mortality rates. Results: The regions show a decline in infant mortality due to the three causes with differences between them. Men and children under a year showed a greater proportion of deaths, with significant differences higher to $80 \%$. An association between the causes of death and the regions of origin of the deceased was found
\end{abstract}


Universidad y Salud

( $p=0,000)$. Conclusion: Although there is decline in the figures of mortality in children under five years old, the implementation of AIEPI has played an important role in controlling these causes of death, but there is no evidence that it has been the main cause for this fall to occur.

Keywords: Malnutrition, child, health, mortality, AIEPI. (Source: DeCS, Bireme).

\section{Introducción}

De acuerdo con la Organización Panamericana de la Salud (OPS), la estrategia sobre la Atención Integrada de Enfermedades Prevalentes de la Infancia, conocida con la sigla AIEPI, es la herramienta para mejorar la salud de la niñez, centrando la atención en el estado general de salud de los menores de cinco años.

Su finalidad es reducir la mortalidad causada por neumonía, diarrea, malaria, tuberculosis, dengue, meningitis, maltrato, trastorno nutricional y enfermedades prevenibles, así como sus complicaciones. Busca también reducir la incidencia y gravedad de los episodios de enfermedades infecciosas que afectan a los niños, para garantizar una adecuada atención, tanto en los servicios de salud, como en el hogar y en la comunidad.

AIEPI se ha incorporado en el Sistema de Salud de Colombia desde 1998 como una estrategia y un enfoque que considera que la buena atención en salud de niños y niñas constituye un derecho; en esa perspectiva el enfoque que se le da es integral, buscando una mayor equidad en la población, fomentando los procesos que estimulan el crecimiento, el desarrollo apropiados y en general, el bienestar de los menores. La estrategia opera a través de tres componentes:

a) Fortalecimiento de los sistemas y servicios de salud.

b) Mejoramiento delas habilidades y prácticas comunitarias, buscando construir y poner en práctica las competencias familiares y sociales.

c) Promoción a partir de 18 prácticas clave, entre las cuales figura como una de las principales, el mejoramiento de las habilidades del personal de salud para la gestión de la estrategia.

AIEPI combina actividades que habitualmente se realizan en forma fragmentada, integrándolas en el nivel de la atención en los servicios de salud. Ha sido implementada en la mayoría de países incluyendo Colombia, impartiendo instrucciones y difundiendo material educativo desde el Ministerio de Salud y Protección Social, con el fin de mejorar la atención en salud. ${ }_{3}$

Al implementarse AIEPI en Colombia, Antioquia fue uno de los departamentos seleccionados para poner en marcha políticas para la protección de la infancia como población vulnerable y específicamente, para la reducción de la morbilidad y mortalidad infantil.

Según la Dirección Seccional de Salud de Antioquia (DSSA), desde su inicio la estrategia se diseñó con los tres componentes que la caracterizan: formación del personal sanitario en el tratamiento de casos, mejoría de los sistemas de salud e implementación de prácticas sanitarias en las familias y comunidades.

La estructuración de esta estrategia como tal se dio desde 1998, pero fue en 2008 cuando, partiendo de la capacitación y difusión realizada, se estructuró como un sistema complejo, se definió su componente económico y se determinó la forma de ejercer el control total y de estructurar todo el sistema.

Con relación a esta estrategia, en Antioquia la DSSA $_{4}$ ha documentado la tendencia a la disminución de las tasas de mortalidad infantil en el Departamento a partir de la década de los noventa, tendencia que es continua y decreciente en el presente siglo; a pesar de presentarse dicha tendencia, la mortalidad infantil evitable sigue siendo un evento de importancia para la salud pública y pilar fundamental de trabajo para todos los países con miras a lograr el desarrollo; entre las principales causas de mortalidad en los menores de cinco años, se encuentran la enfermedad diarreica aguda (EDA), la infección respiratoria aguda (IRA) y la desnutrición, las cuales se describen a continuación debido a su importancia dentro del presento artículo.

Infección Respiratoria Aguda (IRA). Es un conjunto de enfermedades del aparato respiratorio que inician de manera repentina y pueden durar hasta dos semanas; estas son causadas por microorganismos como virus y bacterias; tiene gran frecuencia, no solo en el mundo sino también en Colombia. ${ }_{5}$

En las Américas se registran más de 100.000 defunciones anuales de menores de un año por procesos respiratorios; siendo la complicación más 
destacada la neumonía, que causa el mayor número de muertes en los menores de cinco años de edad. ${ }_{6}$

Enfermedad Diarreica Aguda (EDA). Es una infección del tracto digestivo transmitida por alimentos, agua contaminada o de una persona a otra como resultado de deficiente higiene; puede ser ocasionada por diferentes organismos bacterianos, víricos y parásitos. ${ }_{7}$

En todo el mundo se producen unos 1.700 millones de casos de enfermedades diarreicas cada año, siendo la segunda causa de muerte en niños menores de cinco años; en países en desarrollo, los niños menores de tres años sufren en promedio tres episodios de diarrea al año y cada uno de estos priva al niño de nutrientes necesarios para su crecimiento; la diarrea prolongada puede privar al organismo del agua y las sales necesarias para la supervivencia y causar la muerte por una grave deshidratación y pérdida de líquidos; los niños malnutridos o inmunodeprimidos tienen mayor riesgo de enfermedades diarreicas

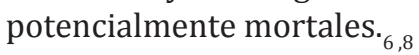

Desnutrición. es un grave problema para la salud pública, principalmente cuando se presenta en los primeros cinco años de vida; su prevalencia se debe a la falta de una alimentación adecuada, con el consiguiente déficit de energía y nutrientes esenciales para llevar una vida sana y activa, lo que acarrea alteraciones en el desarrollo físico y mental, que pueden conducir a enfermedades y en algunos casos a la muerte.

Las causas fundamentales de la desnutrición infantil son complejas y multifactoriales, abarcan el entorno económico, social, político, cultural y físico. Se asocia principalmente a la pobreza, inseguridad alimentaria, falta de acceso a alimentos inocuos, variados y nutritivos y en general a inadecuadas prácticas de lactancia materna exclusiva y de alimentación complementaria.

Las condiciones ambientales como la insalubridad, falta de acceso a agua potable, saneamiento y atención sanitaria y el hacinamiento son otros factores que influyen en el riesgo de padecer desnutrición. 6

En todo el mundo hay cerca de 115 millones de niños con insuficiencia ponderal que contribuye a cerca de un tercio de la mortalidad infantil. El retraso del crecimiento (un indicador de desnutrición crónica) dificulta el desarrollo de 171 millones de niños menores de cinco años. ${ }_{6}$

Estrategia AIEPI. ha sido implementada en varios países del mundo como Bolivia, en donde este enfoque ha permitido identificar las enfermedades no reconocidas habitualmente por las madres o no identificadas por el personal de salud; $;$ en Argentina según Buchbinder, ${ }_{10}$ a pesar de las distintas estrategias (sin mención explícita a ninguna de las implementadas) los indicadores de muertes en niños menores de cinco años se mantienen, situación que se presenta también en México, según Álvarez et al. ${ }_{11}$

En cuanto a Perú, Dávila y Toledo $_{12}$ muestran que no se dan todavía buenos resultados teniendo en cuenta la falta de adiestramiento del personal de salud, baja cobertura de servicios de salud y los escasos recursos de las madres para asistir a las consultas.

Por su parte Benguigui, Bossio y Arias, ${ }_{13}$ señalan que las enfermedades que están bajo la estrategia AIEPI, son las principales causas de muerte en los niños; en cuanto a Antioquia, la DSSA al analizar las muertes de los niños de 1 a 4 años, encuentra que las principales enfermedades causantes son prevenibles, y al comparar cifras de muertes entre el año 2006 y el 2010 , hay eventos que se mantienen aunque cambian de posición. ${ }_{14}$

La mortalidad infantil se ha reducido entre 2005 y 2010 en un 63,9\%, pasando de 13,6 muertes por cada 1.000 nacidos vivos en el 2005 a 8,7 muertes por 1.000 nacidos vivos en el 2010; y comparando esta última, con la meta para el cuatrienio, establecida en el Plan de Desarrollo 2008-2011, de reducir la tasa de mortalidad infantil de 11,58 a 10,29 en el año 2011, se evidencia que a la fecha se ha sobrepasado la meta, ubicándose Antioquia como uno de los departamentos con la tasa de mortalidad infantil más baja, alcanzando una reducción del $45,6 \%$ con respecto de la tasa de mortalidad infantil calculada para Colombia. ${ }_{4}$

Los informes de la Alcaldía de Medellín consignan que los indicadores para dos de las causas de muerte abordadas en AIEPI, son los siguientes: para IRA (equivalente a ERA- Enfermedad Respiratoria Aguda) 
0,13 para 2005, 0,25 en 2006, 0,27 para 2007, 0,19 para 2008 y 0,12 en 2010; para EDA, las tasas de mortalidad en menores de cinco años fueron: 0.09 en 2005, 0.03 en 2006, 0,04 en 2007, 0.03 en 2008, 0.03 en 2009 y 0.00 en $2010 ._{15}$

El presente estudio buscó analizar el comportamiento de la mortalidad en niños menores de cinco años, en las regiones del departamento de Antioquia entre 2002 y 2011, a partir de la implementación de la estrategia AIEPI; mortalidad causada por las principales enfermedades relacionadas con el estado nutricional de los niños y que podrían ser evitadas, según lo informa la OPS, las cuales son infección respiratoria aguda (IRA), enfermedad diarreica aguda (EDA) y desnutrición (DNT) ${ }_{16}$

\section{Materiales y métodos}

Estudio descriptivo retrospectivo con enfoque cuantitativo de fuente secundaria, sobre el comportamiento de las defunciones de menores de cinco años por EDA, IRA y DNT (de acuerdo con los códigos de la Clasificación Internacional de Enfermedades CIE-10), ${ }_{17}$ ocurridas en las regiones del departamento de Antioquia entre 2002 y 2011 antes y después de la implementación de la estrategia AIEPI.

Durante los años en estudio, se registraron 2.270 defunciones en menores de cinco años por dichos eventos; de los cuales el 46,7\% (1.061) fueron por IRA, el 30,7\% (696) por DNT y por EDA el 22,6\% (513). Tanto los registros de defunción, como los de nacidos vivos, fueron tomados del Departamento Administrativo Nacional de Estadísticas (DANE) ${ }_{{ }_{18}}$

En el estudio se incluyeron todas aquellas muertes en menores de cinco años, registradas en la base de datos de defunciones del DANE, entre los años 2002 y 2011, y que su causa básica de muerte hubiera sido IRA, DNT o EDA.

Los datos fueron procesados en el paquete estadístico SPSS versión 21, el análisis univariado se realizó para las variables año, sexo, grupos de edad (menores de 1 año y de 1 a 4 años), región del departamento y causa de defunción; por medio de distribución de frecuencias, proporciones y tasas promedio de mortalidad por cien mil nacidos vivos.
Se realizó análisis bivariado con el fin de identificar posibles asociaciones de las variables utilizando la prueba Chi cuadrado de independencia $\left(\mathrm{x}^{2}\right)$, con un nivel de significación del 5\%.

Este estudio, se hace en torno a la implementación de la estrategia AIEPI en Antioquia, principalmente en los municipios de Medellín, Ciudad Bolívar y Turbo. Las primeras fases de dicha implementación fueron de sensibilización y concertación y hubo participación de las facultades de enfermería y medicina locales; a continuación se dieron cursos de capacitación, seguimiento y expansión de la estrategia AIEPI con asistencia de las compañías aseguradoras, las IPS, y las ONGs.

Este comienzo fue más de tipo académico que práctico, dándose a conocer con la capacitación a los estudiantes de las áreas de la salud de la Universidad de Antioquia en los tres componentes a saber: mejoramiento del personal de salud, fortalecimiento de los servicios de salud y mejoramiento de las prácticas familiares y comunitarias. ${ }_{14}$

\section{Resultados}

Las defunciones en los menores de cinco años, debido a DNT, IRA y EDA en Antioquia, mostraron una tendencia a la disminución durante los años estudiados, siendo el año 2002 el que presentó el mayor porcentaje de muertes, un 14,9\% (339) y el año 2011 el menor con un 5,2\% (119).

Las muertes para cada uno de los eventos mostraron a lo largo de los años un comportamiento más variable, siendo el pico más alto para la IRA en el año 2006 con un 12,6\% (134), para DNT el año 2002 con un 21,4\% (149) y para EDA el año 2003 con un $14,8 \%$ (76), siendo estas diferencias estadísticamente significativas $\left(X^{2}=139,257 ; p=0,000\right)$ (Tabla 1 ).

Para los tres eventos, los hombres registraron más defunciones en cuanto a la edad, fue mayor del $82 \%$ las defunciones en las tres causas en los menores de 1 año, con diferencias significativas por edad $\left(X^{2}=24,218 ; P=0,000\right)$.

En cuanto al comportamiento de las defunciones por regiones, los porcentajes más altos los tuvieron Valle de Aburrá y Urabá (Tabla 1). 
Tabla 1. Distribución proporcional y tasas de mortalidad por IRA, EDA y DNT en menores de cinco años, Departamento de Antioquia, 2002-2011

\begin{tabular}{|c|c|c|c|c|c|c|c|c|c|c|c|c|}
\hline & & IRA & & & utrici & & & EDA & & & Total & \\
\hline Año & $\mathbf{N}$ & $\%$ & Tasa & $\mathbf{N}$ & $\%$ & Tasa & $\mathbf{N}$ & $\%$ & Tasa & $\mathbf{N}$ & $\%$ & Tasa \\
\hline \multicolumn{13}{|l|}{ Sexo } \\
\hline Hombre & 563 & 53,1 & 62,9 & 370 & 53,2 & 41,34 & 286 & 55,8 & 31,95 & 1219 & 53,7 & 136,18 \\
\hline Mujer & 498 & 46,9 & 55,64 & 326 & 46,8 & 36,42 & 227 & 44,2 & 25,36 & 1051 & 46,3 & 117,42 \\
\hline \multicolumn{13}{|l|}{ Región } \\
\hline Bajo Cauca & 109 & 10,3 & 197,5 & 114 & 16,4 & 206,6 & 95 & 18,5 & 172,2 & 318 & 14,1 & 576,3 \\
\hline $\begin{array}{l}\text { Magdalena } \\
\text { Medio }\end{array}$ & 27 & 2,5 & 145,4 & 14 & 2 & 75,38 & 18 & 3,5 & 96,9 & 59 & 2,6 & 317,7 \\
\hline Nordeste & 46 & 4,3 & 179,4 & 18 & 2,6 & 70,2 & 32 & 6,2 & 124,8 & 96 & 4,3 & 374,4 \\
\hline Norte & 51 & 4,8 & 143,6 & 38 & 5,5 & 106,99 & 32 & 6,2 & 90,1 & 121 & 5,4 & 340,7 \\
\hline Occidente & 59 & 5,6 & 228,1 & 37 & 5,3 & 143,05 & 21 & 4,1 & 81,2 & 117 & 5,2 & 452,3 \\
\hline Oriente & 89 & 8,4 & 119,7 & 28 & 4 & 37,65 & 25 & 4,9 & 33,6 & 142 & 6,3 & 190,9 \\
\hline Suroeste & 48 & 4,5 & 112,9 & 37 & 5,3 & 87,06 & 14 & 2,7 & 32,9 & 99 & 4,4 & 233 \\
\hline Urabá & 256 & 24,1 & 234,4 & 180 & 25,9 & 164,81 & 188 & 36,6 & 172,1 & 624 & 27,7 & 571,4 \\
\hline Valle de Aburrá & 367 & 34,6 & 72,2 & 224 & 32,2 & 44,05 & 86 & 16,8 & 16,9 & 677 & 30,0 & 133,1 \\
\hline \multicolumn{13}{|l|}{ Edad } \\
\hline $\begin{array}{l}\text { Menores de } 1 \\
\text { año }\end{array}$ & 872 & 82,2 & 97,4 & 617 & 88,6 & 68,9 & 425 & 82,8 & 97,4 & 1914 & 84,3 & \\
\hline de 1 a 4 años & 189 & 17,8 & 21,1 & 79 & 11,4 & 8,8 & 88 & 17,2 & 9,8 & 356 & 15,7 & \\
\hline Total & 1061 & 46,7 & 118,53 & 696 & 30,7 & 777,56 & 513 & 22,6 & 573,12 & 2270 & 100 & 2536,01 \\
\hline
\end{tabular}

Tasas por cien mil nacidos vivos

Fuente: Departamento Administrativo Nacional de Estadísticas (DANE). Certificados de defunción 2002-2011. Cálculos de las investigadoras. 
La IRA fue el evento que mostró mayor número de muertes, pero fue la desnutrición la que tuvo la tasa de mortalidad más alta, de 777,56 muertes por cada cien mil nacidos vivos, seguido del evento EDA el cual causó durante los años de estudio aproximadamente 573,12 muertes por cada cien mil nacidos vivos; finalmente la IRA mostró una tasa de mortalidad de 118,53 muertes por cada cien mil nacidos vivos.

Para DNT y EDA el año 2002 fue el que mostró la mayor tasa de mortalidad, 159,08 y 80,07 respectivamente por cien mil nacidos vivos; para la IRA fue el año 2006 el que tuvo la tasa más alta $(142,26$ muertes por cada cien mil nacidos vivos). Para IRA y EDA fue el año 2011 el que tuvo más baja tasa de mortalidad $(76,54$ y 23,75 respectivamente por cada cien mil nacidos vivos) y para DNT fue el año 2009 con 41,85 muertes por cada cien mil nacidos vivos.
El riesgo de morir por IRA mostró un comportamiento creciente entre los años 2003 y 2006, llegando en este último a una tasa de 142,26 por cien mil nacidos vivos, en los dos siguientes años se observa una disminución que llega a una tasa de 116,31 muertes por cada cien mil nacidos vivos en el año 2008, aumentando para el siguiente año a 118,57, para nuevamente disminuir hasta el año 2011 en el que llega a tener una tasa de 76,54 muertes por cada cien mil nacidos vivos.

La desnutrición mostró una disminución más constante, comenzando en el año 2002 con una tasa de 159,08, llegando a 45,42 en el año 2007; en el 2008 muestra un marcado aumento, siendo la tasa para este año de 71,32 para disminuir nuevamente en el año $2009(41,85)$ y retomar el aumento hasta el año 2011 en el que la tasa fue de 56,74, todas las tasas dadas por cien mil nacidos vivos. Para EDA se observó un comportamiento más inestable, aumentando y disminuyendo durante los años estudiados (Gráfico 1).

\section{Gráfico 1. Tasas de mortalidad por desnutrición, IRA y EDA en menores de cinco años, según año. Departamento de Antioquia, 2002-2011}

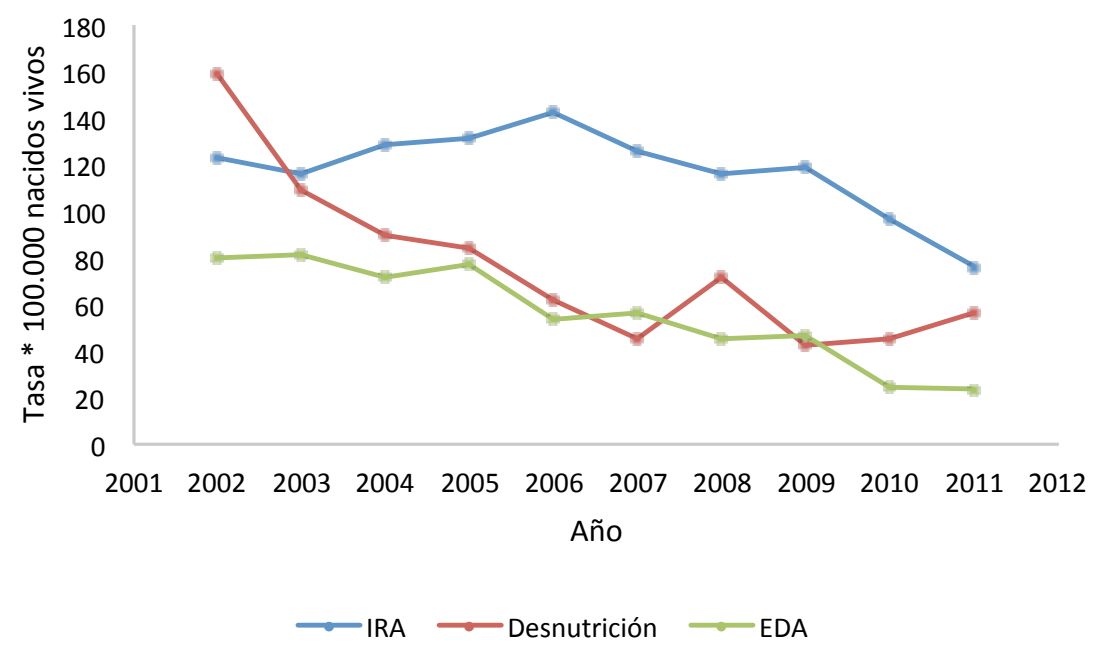

Fuente: Departamento Administrativo Nacional de Estadísticas (DANE). Certificados de defunción 2002-2011. Elaboración propia.

En cuanto a cada una de las subregiones del departamento, en Bajo Cauca y Urabá se registró el mayor riesgo de morir por estas causas; para IRA la tasa más alta se presentó en la región de Urabá con una tasa de 234,40 muertes por cada cien mil nacidos vivos, seguida de región Occidente con una tasa de 228,11 por cada cien mil nacidos vivos; por DNT la mayor tasa se observó en el Bajo Cauca con una tasa de 206,60 muertes por cada cien mil nacidos vivos y en Urabá con una tasa de 164,81 por cada cien mil nacidos vivos; finalmente para el evento EDA, las mayores tasas también se observaron en Bajo Cauca y Urabá con tasas de 172,17 y 172,14 respectivamente por cada cien mil nacidos vivos (Gráfico 2). 
Gráfico 2. Tasas de mortalidad por desnutrición, IRA y EDA en menores de 5 años en cada subregión de Antioquia, 2002-2011

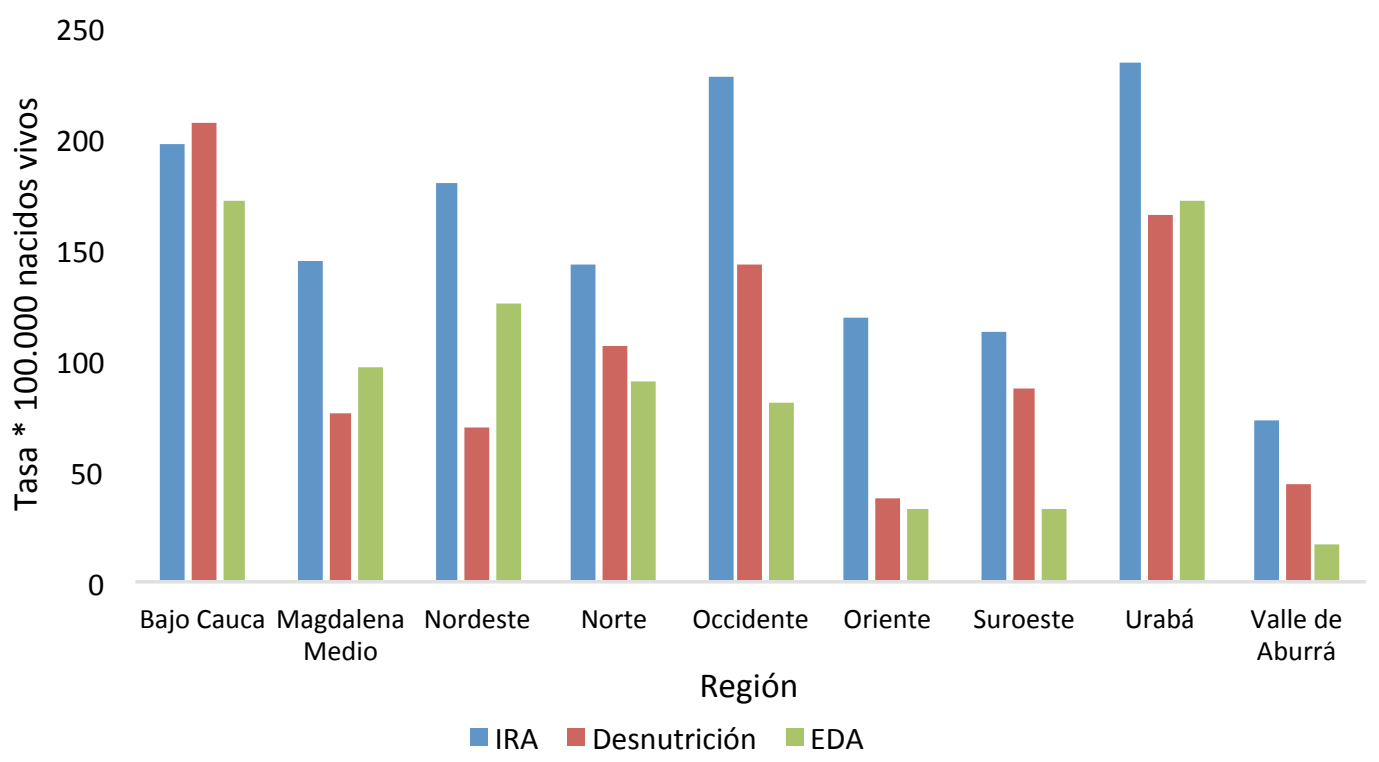

Fuente: Departamento Administrativo Nacional de Estadísticas (DANE). Certificados de defunción 2002-2011.

Para los tres eventos, fueron los hombres quienes mostraron mayor tasa de mortalidad en menores de cinco años; como es el caso de IRA, siendo esta de 62,9 muertes por cada cien mil hombres nacidos; de igual forma para las mujeres, este fue el evento que mostró mayor tasa de mortalidad (55,64 muertes por cada cien mil mujeres nacidas vivas).

En cuanto al grupo de edad, para los tres eventos la tasa fue mucho más alta en los menores de un año en comparación con los de 1 a 4 años, siendo estas tasas para el total, de 84,3 y 15,7 respectivamente, por cada cien mil nacidos vivos; en los menores de un año, la menor tasa la tuvo desnutrición, la cual fue de 68,9 muertes por cada cien mil nacidos vivos, IRA y EDA tuvieron tasas de 97,4 muertes por cada cien mil nacidos vivos; en los niños de 1 a 4 años, la mayor tasa presentada fue por IRA con 21,1, seguida de EDA con 9,8 y desnutrición con 8,8 (todas las tasas por cien mil nacidos vivos).

\section{Discusión}

La estrategia AIEPI es actualmente una de las principales herramientas utilizadas para mejorar el estado de salud de los niños menores de cinco años, enfocándose en la detección precoz y tratamiento de enfermedades que podrían no ser identificadas fácilmente por padres y personal de salud. ${ }_{2}$ Esta estrategia se comienza a implementar principalmente a través de la capacitación del personal de salud y de la educación a la comunidad; ha sido impulsada en el departamento de Antioquia a través de la Gobernación y de la Secretaría Seccional de Salud y Protección Social de Antioquia, buscando con esto mejorar la salud y calidad de vida de los niños. ${ }_{19}$ Como se aclaró inicialmente en la metodología, el análisis de estos resultados se hace principalmente alrededor de la implementación de la estrategia AIEPI en Antioquia.

En el período de gobierno 2001-2003 “Una Antioquia Nueva", no hubo desarrollo específico de la estrategia, según se consigna en la Dirección Seccional de Salud Gobernación de Antioquia, 2008 lo cual se ve reflejado en los resultados de esta investigación, en la que 
durante los años 2002 y 2003 se observan los más altos porcentajes y tasas de mortalidad en menores de cinco años, debido a tres de los eventos en los que se enfoca la estrategia (IRA, DNT y EDA) ${ }_{14}$

Luego, en el período de gobierno 2004-2007 "Antioquia Nueva: un hogar para la vida", se incluye la estrategia AIEPI y se armoniza con el Plan de Desarrollo Departamental de manera que tuviera factibilidad financiera. Se inscribe en el Banco de Proyectos de Antioquia del Departamento Administrativo de Planeación, el cual puede consultarse con el nombre de "Implementación de la Estrategia de Atención Integrada a las Enfermedades Prevalentes de la Infancia (AIEPI), departamento de Antioquia, cuatrienio 2004-2007", por un valor de $\$ 1934,4$ millones de pesos.

Este convenio se ejecuta con la participación de la Universidad de Antioquia, la Universidad Pontificia Bolivariana y ECOSESA. Agrega el informe que: "realizó la inducción a 86 municipios, para una cobertura del 68\%; capacitación en organización local a 78 municipios (62\%); talleres de seguimiento a 86 municipios (64\%); capacitación en AIEPI Clínico a 121 municipios correspondiente a una cobertura del 97\% y capacitación en el componente comunitario a 93 municipios (74\%); para estos años.

Este estudio observó que los porcentajes y tasas de mortalidad difieren de acuerdo al evento, observándose un importante aumento para IRA entre 2004 y 2006 , pasando de una tasa de 128,22 a 142,26 muertes por cien mil nacidos vivos; la desnutrición y EDA, mostraron disminución en estos años, en comparación a los dos años analizados anteriormente, la tasa de mortalidad por desnutrición, que para el año 2003 había sido de 109,42, se redujo a 90,38 en el año 2004, disminuyendo en los siguientes años, hasta llegar a 45,42 en el 2007, siendo estas tasas de mortalidad por cada cien mil nacidos vivos; para EDA, la situación es muy similar, pasando de una tasa de 80,73 en el año 2003 a 71,47 en el 2004, la cual para el año 2007 se presenta más baja aun $(56,24)$, todas las tasas dadas por cien mil nacidos vivos. ${ }_{14}$

En el período 2008-2011 se armonizaron los componentes de AIEPI con el Plan Nacional de Salud Pública 2007-2010 dándole prioridad a la primera infancia y se integraron con el Plan de Desarrollo departamental de Antioquia para la reducción de la incidencia de las enfermedades y problemas de salud de los menores, el crecimiento y desarrollo durante los primeros años de vida.

La celebración de un convenio interadministrativo Universidad de Antioquia - grupo Nacer tuvo como objetivo extender los beneficios a los 125 municipios y en desarrollo de este se realizó la capacitación y seguimiento de la estrategia AIEPI en lo que tiene que ver con el componente clínico con enfermeros y enfermeras, así como con médicos y en lo que tiene que ver con la historia clínica. Estas capacitaciones, además del material académico necesario, incluyeron el manejo del libro AIEPI clínico, el cuadro de procedimientos y formatos de histórica clínica para menores de dos meses, de dos meses a cinco años y gestantes, así como un plan de acción de seguimiento a los pacientes. ${ }_{14}$

Se apoyó a las instituciones en la elaboración de una línea de base para el personal capacitado, y se fortaleció el desarrollo de actividades que articularan la estrategia AIEPI con un acompañamiento de nueve talleres regionales de capacitación para fortalecer el propio trabajo y para la construcción de un plan de acción comunitario.

Se implementó igualmente, un apoyo para la vigilancia epidemiológica de la mortalidad por IRA y EDA, puesto que el objetivo primordial de la adecuada implementación del AIEPI y las ejecuciones para alcanzarlo debían reflejarse en la disminución de la mortalidad, lo cual, según los resultados de la presente investigación, se comienza a materializar, observándose una importante disminución en las tasas de mortalidad de los tres eventos analizados; durante estos cuatro años; la disminución que mostró la mortalidad por IRA que para el año 2008 fue de 116,31, tuvo únicamente un pico que reflejo aumento, en el año 2009 (118,57), reduciendo la cifra nuevamente en los dos siguientes años, hasta llegar a 76,54 muertes por cada cien mil nacidos vivos en el año 2011.14

La tasa de mortalidad por desnutrición, mostró una gran caída entre el año 2008 (71,32), y el año 2009 $(41,85)$; luego de esta disminución se observó un leve aumento para el año $2010(44,91)$ y el año 2011 $(56,74)$, aumento que a pesar de ser importante, no supera las tasas que se tenían en años anteriores. 
La tasa de mortalidad por EDA, que para el año 2007 había sido de 56,24, comienza a disminuir en el año 2008 y finalmente llega a 23,75 para el 2011. El comportamiento de la mortalidad que según los resultados de este estudio se presentan a partir del año 2008 en el que se comienza la armonización de los componentes del AIEPI y el Plan Nacional de Salud Pública, permitieron apreciar, que aunque las tasas continúan siendo altas, al ser comparadas con las de los años anteriores, son inferiores, lo cual, al ser observado desde el punto de vista del impacto de la estrategia, muestra resultados positivos y una tendencia a mejorar.

Para el 2008 el gobierno departamental pone en marcha un programa integrado de AIEPI, MANA (Mejoramiento Alimentario y Nutricional de Antioquia) y APS (Atención Primaria en Salud) con el fin de "aunar esfuerzos, potenciar recursos y tener un mayor impacto para dar respuesta a problemas identificados" y en estos problemas se encuentran considerados los riesgos de "padecer una enfermedad evitable antes de los cinco años (IRA, EDA, DNT, entre otras)". ${ }_{14}$

De acuerdo con el DSSA $_{4}$ la distribución porcentual de la mortalidad en menores de cinco años por sexo para el año de base 2006, fue mayor en niñas con un $55,2 \%$ (838) con respecto a los niños con un 44,8\% (681). Para el año 2010, el sexo femenino fue el más aportante con $56,2 \%$ (545) respecto del sexo masculino con un $43,4 \%$ (421). Al revisar la frecuencia de muertes en menores de cinco años según régimen de afiliación, se observa que los vinculados al régimen subsidiado son los que más aportan, lo que puede estar evidenciando inequidades en el acceso y calidad de la prestación de los servicios de salud.

Durante el 2010, se observaron 128 casos sin aseguramiento y 53 casos sin dato de afiliación; indicando la prioridad de afiliación de los niños y las niñas menores de cinco años, así como la intensificación del seguimiento al correcto diligenciamiento del certificado de defunción.

En el municipio de Medellín se han aplicado los lineamientos de la estrategia desde el 2008, aunque la guía operativa sólo se publica en el año 2011. Los resultados de muertes infantiles por DNT, IRA, EDA en el período entre 2002 y 2011 con cifras tomadas del registro de defunciones del DANE, efectivamente muestran el decrecimiento de las cifras de mortalidad en neonatos, niños y niñas menores de 5 años, que según las cifras nacionales y departamentales, comienza en los años finales de la última década del siglo XX y se da a lo largo de los años trascurridos entre 2000 y 2011. Este trabajo, confirma la disminución que se ha venido presentando desde el año 2002 y los resultados pueden verse desagregados por regiones. Las cifras de la tendencia departamental de la DSSA muestran el descenso de 2002 en adelante y lo confirman los hallazgos por regiones, a excepción del Magdalena Medio.

Es creciente la cifra de muertes en el Magdalena Medio cuando la causa son las infecciones respiratorias. Los datos del departamento de Antioquia, no coinciden con los de la OPS (2010) que señala al departamento de Antioquia como uno de los que presentan falencias en la implementación del AIEPI. $_{3}$

En cuanto a los resultados y hallazgos, se encuentra que los más altos índices de presencia de enfermedades infantiles por regiones, se encuentran en el Magdalena Medio, Nordeste y Suroeste, por comparación con las restantes subregiones del departamento de Antioquia. Sin embargo, el comportamiento de las variables presenta una tendencia a la baja que coincide con la tendencia general más reciente en el departamento.

Los indicadores de IRA, DNT y EDA han presentado descensos porcentuales importantes. Para IRA se muestran cifras de $10,8 \%$ para 2002 , y de $5,5 \%$ en 2011; DNT presenta un descenso desde 2002, aunque muestra un incremento en 2008 de 3,3\% y desciende nuevamente el año 2009 en 4,1\%, con una mayor mortalidad en hombres.

Los datos confirman los resultados de la Dirección Seccional de Salud en lo que tiene que ver con las regiones, pero no pudieron contrastarse con los del municipio de Medellín, por cuanto se trabajaron las cifras regionales aportadas por el DANE y no están desagregadas las de Medellín, siendo las cifras del municipio las que aportan las investigaciones propias de este $_{4}$

Se observa una mejora cualitativa de la salud de niños y niñas menores de cinco años, pero no puede 
afirmarse que la aplicación de la estrategia AIEPI haya tenido un impacto definitivo en las regiones, pues tendría que observarse una reducción drástica a partir de 2007, cuando la Gobernación de Antioquia realiza una planeación de la estrategia en términos de una política departamental.

Pese a que la estrategia ha tenido gran importancia en el conjunto de medidas gubernamentales, en los resultados obtenidos no se observa una mejoría tan marcada como la que se esperaría al implementarse AIEPI; los resultados de las regiones muestran cifras altas de mortalidad en menores de cinco años, especialmente por IRA; aunque el programa elaborado en 2007 incluye el monitoreo, el seguimiento y la evaluación del mismo, no se encontró la documentación que se refiere a estos temas.

La estrategia AIEPI en el departamento de Antioquia, ha tenido realmente aplicación continua a partir del año 2008, ya que antes de este año, el enfoque estuvo dirigido principalmente a desarrollar talleres de capacitación del personal de la salud; por lo tanto se puede decir, que aunque la implementación de esta estrategia en Antioquia ha mostrado un desempeño favorable, la reducción que se ha presentado en las tasas de mortalidad no pueden atribuirse en definitiva a esta, ya que durante los años 2002 y 2007 la aplicación de la estrategia tuvo como objetivo el delineamiento de las estrategias jurídicas y contractuales y la capacitación de personal y en menor medida se enfocó en las acciones para el control de los eventos; por lo tanto, es a partir del año 2008 que se comienza la aplicación de la estrategia como tal.

En cuanto a las limitaciones que presenta el estudio están dadas por el hecho de no encontrar la evaluación mencionada que posibilitaría contrastar los resultados de manera más amplia. Además, porque los resultados corresponden a regiones y no a los municipios pertenecientes a cada una. Una mayor exhaustividad en estos puede lograrse cuando se tomen los datos locales y puedan contratarse los de los distintos municipios por región y sus resultados con las tendencias nacionales.

\section{Conclusiones}

Los resultados de la investigación, permitieron observar la disminución que han presentado las tasas de mortalidad en menores de cinco años, por IRA, EDA y DNT, esto durante los años 2002 y 2011. En cuanto a las regiones, se observaron ascensos y descensos a lo largo del periodo, con tasas que tienden a su reducción; en las regiones de Bajo Cauca, Norte, Magdalena Medio, Occidente y Oriente las tasas a pesar de que muestran reducción, se observa un pequeño incremento para los años 2010 y 2011. La disminución estaba contemplada dentro de los objetivos de la estrategia AIEPI que se concluye que a pesar de que las tasas de mortalidad por dichos eventos, en menores de cinco años continua siendo alta, desde que se ha venido implementando esta estrategia en el departamento de Antioquia, los indicadores de mortalidad si han mostrado cambios que tienen a la disminución en el transcurso de los años.

Mirar hacia temporalidades como la analizada, constituyó una reflexión sobre la experiencia producida y permite pensar en una sistematización de los resultados teniendo como objetivo los municipios de cada región, lo que sería un estudio importante que posibilitaría ampliar los rangos del conocimiento local, con miras a una aplicación, tanto extensiva como intensiva de los componentes de la estrategia AEIPI con el objetivo de mejorar la salud infantily reducir los índices de mortalidad. Finalmente, es evidente la necesidad de una acción continua de todos los organismos de salud de manera que las cifras puedan descender significativamente en toda la región, por lo cual se propone a las autoridades de salud del departamento que se extienda la capacitación en la estrategia AIEPI a todo el personal, no solo al que atiende en hospitales y clínicas, sino al que tiene un mayor contacto con los niños y niñas enfermos: los centros y puestos de salud y en especial todos los servicios de urgencias del departamento de Antioquia.

\section{Referencias}

1. Organización Mundial de la Salud. Atención Integrada a las Enfermedades Prevalentes de la Infancia (AIEPI). Salud de la madre, el recién nacido, del niño y del adolescente. [citado 5 de octubre de 2014]. Disponible 
en: http://www.who.int/maternal_child_adolescent/ topics/child/imci/es/

2. Organización Panamericana de la Salud. Sobre la atención integrada de enfermedades prevalentes de la infancia AIEPI [Internet]. Disponible en: http:// www.paho.org/col/index.php?option $=$ com_cont ent \&view=article\&id=1552: atencion-integradade-enfermedades-prevalentes-de-la-infanciaaiepi\&Itemid $=476$

3. República de Colombia, Ministerio de la Protección Social, Organización Panamericana de la Salud. Atención integrada a las enfermedades prevalentes de la infancia, cuadro de procedimientos. Colombia: Minprotección, OPS; 2010.

4. Dirección Seccional de Salud de Antioquia. Análisis de situación de salud en Antioquia. Mortalidad. Medellín: DSSA; 2010.

5. Ministerio de Salud y Protección Social. ¿Qué es una infección respiratoria aguda? [Internet]. Infecciones Respiratorias Agudas (IRA). [citado 4 de septiembre de 2014]. Disponible en: http://www.minsalud.gov. co/salud/Paginas/Infecciones-Respiratorias-Agudas(IRA).aspx

6. The United Nations Children's Fund, World Health Organization. Pneumonia the forgotten killer of children. New York: UNICEF, WHO; 2006.

7. Ministerio de Salud República de Argentina. Enfermedades diarreicas [Internet]. [citado 23 de junio de 2015]. Disponible en: http://www.msal.gov.ar/ index.php/programas-y-planes/133-enfermedadesdiarreicas

8. Organización Mundial de la Salud. Enfermedades diarreicas [Internet]. Centro de prensa. Nota descriptiva No 330. 2013 [citado 23 de junio de 2015]. Disponible en: http://www.who.int/mediacentre/ factsheets/fs330/es/

9. Zamora A, Cordero D, Mejía M. Evaluación de la estrategia "Atención integrada a las enfermedades prevalentes de la infancia» (AIEPI) en servicios de salud. Bolivia. Rev. Chil. Pediatr. 2002;73 (2); 184-191, 2002.

10. Buchbinder M. Mortalidad infantil y desigualdad socioeconómica en la Argentina. Tendencia temporal. Arch Argent Pediatr. 2008;106(3):212-218.

11. Álvarez G, Lara F, Harlow S, Deman C. Mortalidad infantil y marginación urbana: análisis espacial. Revista Panamericana de Salud Pública. 2009; 26(1).
12. Dávila M, Toledo J. Los diez primeros años de AIEPI en el Perú. Manejo de casos de infecciones respiratorias agudas en establecimientos del Ministerio de Salud. Lima: OPS; 2006.

13. Benguigui Y, Bossio J, Arias S. Aiepi en las Américas. Enfermedades prevalentes en la infancia en las Américas. Washintgton: OPS; 2008.

14. Gobernación de Antioquia, Organización Panamericana de la Salud, Dirección Seccional de Salud de Antioquia. Gestión de las experiencias MANA, APS, AIEPI. Antioquia hacia la protección social y el desarrollo humano. Medellín; 2008.

15. Alcaldía de Medellín. Diagnóstico situacional de infancia y adolescencia en el municipio de Medellín. 2012.

16. Organización Panamericana de la Salud. Niñez protegida con estrategia AIEPI [Internet]. AIEPI. 2012 [citado 30 de junio de 2015]. Disponible en: http:// www.paho.org/col/index.php?option=com_content\&v iew=article\&id=1492:ninez-protegida-con-estrategiaaiepi\&catid $=755 \&$ Itemid $=476$

17. Organización Panamericana de la Salud. Clasificación estadística internacional de enfermedades y problemas relacionados con la salud. Décima revisión. Washington, D.C: OPS; 1995.

18. Departamento Administrativo Nacional de Estadística (DANE). Estadísticas por tema [Internet]. 2013 [citado 10 de octubre de 2014]. Disponible en: https://www.dane.gov.co/index.php?option=com search\&searchword=causas

19. Gobernación de Antioquia. AIEPI se multiplica [Internet]. Noticias. 2011 [citado 26 de junio de 2015]. Disponible en: http://www.antioquia.gov.co/index. php/149-2011-octubre/6157-aiepi-se-multiplica 